\title{
Observation of the $\Omega_{b}^{-}$and Measurement of the Properties of the $\Xi_{b}^{-}$and $\Omega_{b}^{-}$Baryons at CDF
}

\author{
Patrick LUKENS* \\ Fermilab \\ E-mail: ptl@fnal.gov
}

We report the observation of the bottom, doubly-strange baryon $\Omega_{b}^{-}$through the decay chain $\Omega_{b}^{-} \rightarrow J / \psi \Omega^{-}$, where $J / \psi \rightarrow \mu^{+} \mu^{-}, \Omega^{-} \rightarrow \Lambda K^{-}$, and $\Lambda \rightarrow p \pi^{-}$, using $4.2 \mathrm{fb}^{-1}$ of data from $p \bar{p}$ collisions at $\sqrt{s}=1.96 \mathrm{TeV}$, and recorded with the Collider Detector at Fermilab. A signal is observed whose probability of arising from a background fluctuation is $4.0 \times 10^{-8}$, or 5.5 Gaussian standard deviations. The $\Omega_{b}^{-}$mass is measured to be $6054.4 \pm 6.8$ (stat.) \pm 0.9 (syst.) $\mathrm{MeV} / \mathrm{c}^{2}$. The lifetime of the $\Omega_{b}^{-}$baryon is measured to be $1.13_{-0.40}^{+0.53}$ (stat.) \pm 0.02 (syst.) ps. In addition, for the $\Xi_{b}^{-}$baryon we measure a mass of $5790.9 \pm 2.6$ (stat.) \pm 0.8 (syst.) $\mathrm{MeV} / \mathrm{c}^{2}$ and a lifetime of $1.56_{-0.25}^{+0.27}$ (stat.) \pm 0.02 (syst.) ps. Under the assumption that the $\Xi_{b}^{-}$and $\Omega_{b}^{-}$are produced with similar kinematic distributions to the $\Lambda_{b}^{0}$ baryon, we find $\frac{\sigma\left(\Xi_{b}^{-}\right) \mathscr{B}\left(\Xi_{b}^{-} \rightarrow J / \psi \Xi^{-}\right)}{\sigma\left(\Lambda_{b}^{0}\right) \mathscr{B}\left(\Lambda_{b}^{0} \rightarrow J / \psi \Lambda\right)}=$

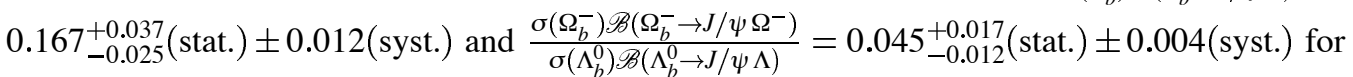
baryons produced with transverse momentum in the range of $6-20 \mathrm{GeV} / c$.

European Physical Society Europhysics Conference on High Energy Physics, EPS-HEP 2009, July 16 - 222009

Krakow, Poland

*Speaker.

${ }^{\dagger}$ On behalf of the CDF Collaboration. 


\section{Introduction}

In this paper, we report the observation of a heavy baryon and the measurement of its mass, lifetime, and relative production rate compared to the $\Lambda_{b}^{0}$ production [1]. The decay properties of this state are consistent with the weak decay of a $b$-baryon. We interpret our result as the observation of the $\Omega_{b}^{-}$baryon $(|s s b\rangle)$.

This $\Omega_{b}^{-}$observation is made in $p \bar{p}$ collisions at a center of mass energy of $1.96 \mathrm{TeV}$ using the Collider Detector at Fermilab (CDF II), through the decay chain $\Omega_{b}^{-} \rightarrow J / \psi \Omega^{-}$, where $J / \psi \rightarrow$ $\mu^{+} \mu^{-}, \Omega^{-} \rightarrow \Lambda K^{-}$, and $\Lambda \rightarrow p \pi^{-}$. Charge conjugate modes are included implicitly. Mass, lifetime, and production rate measurements are also reported for the $\Xi_{b}^{-}$, through the similar decay chain $\Xi_{b}^{-} \rightarrow J / \psi \Xi^{-}$, where $J / \psi \rightarrow \mu^{+} \mu^{-}, \Xi^{-} \rightarrow \Lambda \pi^{-}$, and $\Lambda \rightarrow p \pi^{-}$. The production rates of both the $\Xi_{b}^{-}$and $\Omega_{b}^{-}$are measured with respect to the $\Lambda_{b}^{0}$, which is observed through the decay chain $\Lambda_{b}^{0} \rightarrow J / \psi \Lambda$, where $J / \psi \rightarrow \mu^{+} \mu^{-}$, and $\Lambda \rightarrow p \pi^{-}$. These measurements are based on a data sample corresponding to an integrated luminosity of $4.2 \mathrm{fb}^{-1}$.

The strategy of the analysis presented here is to demonstrate the reconstruction and property measurements of the $\Xi_{b}^{-}$and $\Omega_{b}^{-}$as natural extensions of measurements that can be made on better known $b$-hadron states obtained in the same data. All measurements made here are performed on the $B^{0} \rightarrow J / \psi K^{*}(892)^{0}, K^{*}(892)^{0} \rightarrow K^{+} \pi^{-}$final state, to provide a large sample for comparison to other measurements. The decay modes $B^{0} \rightarrow J / \psi K_{s}^{0}, K_{s}^{0} \rightarrow \pi^{+} \pi^{-}$and $\Lambda_{b}^{0}$ are also used as reference processes.

\section{Particle Reconstruction Methods}

The analysis presented here is based on events recorded with a trigger that is dedicated to the collection of a $J / \psi \rightarrow \mu^{+} \mu^{-}$sample. The analysis of the data begins with a selection of well-measured $J / \psi \rightarrow \mu^{+} \mu^{-}$candidates. This data sample provides approximately $2.9 \times 10^{7} \mathrm{~J} / \psi$ candidates, measured with an average mass resolution of $\sim 20 \mathrm{MeV} / c^{2}$.

The reconstruction of $K_{s}^{0}, K^{*}(892)^{0}$, and $\Lambda$ candidates uses all tracks with $p_{T}>0.4 \mathrm{GeV} / c$ that are not associated with muons in the $J / \psi$ reconstruction. Candidate selection for these neutral states is based upon the mass calculated for each oppositely charged track pair, which is required to fall within $\pm 30, \pm 20$, and $\pm 9 \mathrm{MeV} / c^{2}$ of the nominal mass for the $K^{*}(892)^{0}, K_{s}^{0}$, and $\Lambda$, respectively. Backgrounds to the $K_{s}^{0}$ and $\Lambda$ are reduced by requiring the flight distance of the $K_{s}^{0}$ and $\Lambda$ with respect to the primary vertex to be greater than $1.0 \mathrm{~cm}$. Approximately $3.6 \times 10^{6} \Lambda$ candidates are found with with $p_{T}(\Lambda)>2.0 \mathrm{GeV} / c$.

For events that contain a $\Lambda$ candidate, the remaining tracks are assigned the pion or kaon mass, and $\Lambda \pi^{-}$or $\Lambda K^{-}$combinations are identified that are consistent with the decay process $\Xi^{-} \rightarrow \Lambda \pi^{-}$ or $\Omega^{-} \rightarrow \Lambda K^{-} \cdot p_{T}\left(K^{-}\right)>1.0 \mathrm{GeV} / c$ is required for our $\Omega^{-}$sample, which reduces the combinatorial background by $60 \%$, while reducing the $\Omega^{-}$signal predicted by our Monte Carlo simulation by $25 \%$. In addition, the flight distance of the $\Lambda$ candidates with respect to the reconstructed decay vertex of the $\Xi^{-}\left(\Omega^{-}\right)$, and the flight distance from the primary vertex of the $\Xi^{-}$and $\Omega^{-}$candidates is required to exceed $1.0 \mathrm{~cm}$. Kinematic reflections are removed from the $\Omega^{-}$sample by requiring that the combinations consistent with $\Xi^{-}$decay, when the candidate $K^{-}$track is assigned the mass of the $\pi^{-}$. Any ambiguities for the proper track assignments of the hadrons are resolved examining 
the $P\left(\chi^{2}\right)$ of the vertex fits. Approximately $41000 \Xi^{-}$and $3500 \Omega^{-}$candidates are found in this data sample. $\Lambda \pi^{-}$or $\Lambda K^{-}$combinations within \pm 9 and $\pm 8 \mathrm{MeV} / c^{2}$ of the nominal $\Xi^{-}$and $\Omega^{-}$ masses are selected for $b$-hadron reconstruction.

The reconstruction of $b$-hadron candidates uses the same method for each of the states reconstructed for this analysis. The $K$ and hyperon candidates are combined with the $J / \psi$ candidates by fitting the full four-track or five-track state with constraints appropriate for each decay topology and intermediate hadron state. Specifically, the $\mu^{+} \mu^{-}$mass is constrained to the nominal $J / \psi$ mass [2], and the neutral $K$ or hyperon candidate is constrained to originate from the $J / \psi$ decay vertex. In addition, the fits that include the charged hyperons constrain the $\Lambda$ candidate tracks to the nominal $\Lambda$ mass [2], and the $\Xi^{-}$and $\Omega^{-}$candidates to their respective nominal masses [2]. For our final sample, $b$-hadron candidates are required to have $p_{T}>6.0 \mathrm{GeV} / c$ and the neutral $K$ or hyperon to have $p_{T}>2.0 \mathrm{GeV} / c$. The promptly-produced combinatorial background is suppressed by rejecting candidates with low proper decay time, and spurious combinations are rejected by placing a requirement on the final state impact with respect to the beamline.
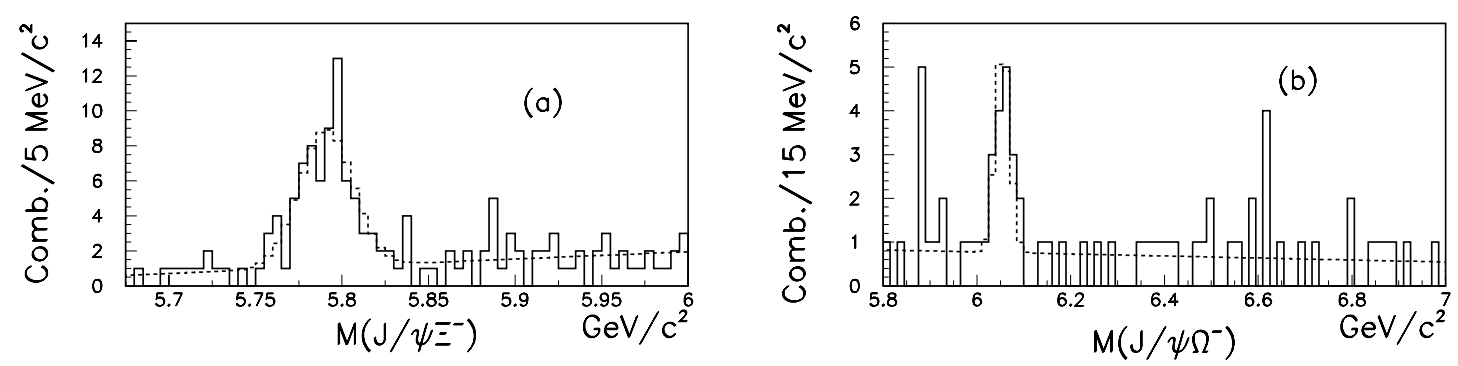

Figure 1: The invariant mass distributions of (a) $J / \psi \Xi^{-}$and (b) $J / \psi \Omega^{-}$combinations for candidates with $c t>100 \mu \mathrm{m}$. The projections of the unbinned mass fit are indicated by the dashed histograms.

\section{Observation of the Decay $\Omega_{b}^{-} \rightarrow J / \psi \Omega^{-}$}

The $J / \psi \Omega^{-}$mass distribution with $c t>100 \mu \mathrm{m}$ is shown in Fig. $1 \mathrm{~b}$. The significance of the structure seen in the $J / \psi \Omega^{-}$mass distribution is evaluated with a simultaneous fit to mass and lifetime information which is maximized for two different conditions. The first maximization allows all parameters to vary in the fit. The second calculation fixes the signal fraction to 0.0. The value of $-2 \ln \mathscr{L}$ obtained for the null hypothesis is higher than the value obtained for the fully varying calculation by 37.3 units. We interpret this as equivalent to a $\chi^{2}$ with three degrees of freedom, which has a probability of occurrence of $4.0 \times 10^{-8}$, or a $5.5 \sigma$ fluctuation. Consequently, we interpret the $J / \psi \Omega^{-}$mass distributions shown in Fig. 1b to be the observation of a weakly decaying resonance, with a width consistent with the detector resolution. We treat this resonance as observation of the $\Omega_{b}^{-}$baryon through the decay process $\Omega_{b}^{-} \rightarrow J / \psi \Omega^{-}$.

\section{4. $\Xi_{b}^{-}$and $\Omega_{b}^{-}$Property Measurements}

To reduce the background to $b$-hadrons due to prompt production, a $c t>100 \mu$ m requirement is placed on all candidates for inclusion in the mass measurements. The mass distributions of the 
candidates are shown in Fig. 1, along with projections of the fit function. The results of this fit are listed in Table 1. Systematic uncertainties for the $\Xi_{b}^{-}$and $\Omega_{b}^{-}$masses are largely driven by our $B^{0}$ mass measurements, and are estimated to be 0.8 and $0.9 \mathrm{MeV} / c^{2}$, respectively.

The lifetime of $b$-hadrons is measured in this analysis by a technique that is insensitive to the detailed lifetime characteristics of the background. This allows a lifetime calculation to be performed on a relatively small sample, since a large number of events is not needed for a background model to be developed. The data are binned in $c t$, and the number of signal candidates in each $c t$ bin is compared to the value that is expected for a particle with a given lifetime and measurement resolution. The estimates of the systematic uncertainties are obtained from the the $b$ lifetime measurements. The results of the fits for the lifetimes of the baryons and reference samples are listed in Table 1.

A further goal of this analysis is to measure the production rates of the $\Xi_{b}^{-}$and $\Omega_{b}^{-}$, relative to the more plentiful $\Lambda_{b}^{0}$, where we measure ratios of cross section times branching fractions. The acceptances and efficiencies of the three baryons states are obtained as a function of $p$ from the simulation of the detector. We use the observed $p_{T}$ distribution of $\Lambda_{b}^{0}$ production to obtain the total efficiency for the $\Xi_{b}^{-}$and $\Omega_{b}^{-}$states. The yields of the baryons are obtained from the lifetime fits, and are listed in Table 1, along with our measurements of the relative production rates for the $\bar{b}$ and $\Omega_{b}^{-}$.

Table 1: Properties obtained for $b$-hadrons.

\begin{tabular}{ccccc}
\hline \hline Resonance & Candidates & Mass $\left(\mathrm{MeV} / c^{2}\right)$ & $c \tau(\mu \mathrm{m})$ & $\frac{\sigma \mathscr{B}}{\sigma\left(\Lambda_{b}^{0}\right) \mathscr{B}\left(\Lambda_{b}^{0} \rightarrow J / \psi \Lambda\right)}$ \\
\hline$B^{0}\left(J / \psi K^{*}(892)^{0}\right)$ & $17520 \pm 305$ & $5279.2 \pm 0.2$ & $453 \pm 6$ & - \\
$B^{0}\left(J / \psi K_{s}^{0}\right)$ & $9424 \pm 167$ & $5280.2 \pm 0.2$ & $448 \pm 7$ & - \\
$\Lambda_{b}^{0}$ & $1934 \pm 93$ & $5620.3 \pm 0.5$ & $472 \pm 17$ & - \\
$\Xi_{b}^{-}$ & $66_{-9}^{+14}$ & $5790.9 \pm 2.6 \pm 0.8$ & $468_{-74}^{+82} \pm 0.06$ & $0.167_{-0.025}^{+0.037} \pm 0.012$ \\
$\Omega_{b}^{-}$ & $16_{-4}^{+6}$ & $6054.4 \pm 6.8 \pm 0.9$ & $340_{-120}^{+160} \pm 0.04$ & $0.045_{-0.012}^{+0.017} \pm 0.004$ \\
\hline \hline
\end{tabular}

\section{Conclusions}

In conclusion, we have used data collected with the CDF II detector at the Tevatron to observe a signal of $16_{-4}^{+6} \Omega_{b}^{-}$candidates, with a significance equivalent to $5.5 \sigma$ when combining both mass and lifetime information. The mass, lifetime and relative production rates of the $\Omega_{b}^{-}$and $\Xi_{b}^{-}$are measured with the best level of precision that has been obtained.

\section{References}

[1] T. Aaltonen et al. (CDF Collaboration), arXiv:0905.3123 (2009).

[2] C. Amsler et al. (Particle Data Group), Phys. Lett. B 667, 1 (2008). 